\title{
Interpreting first-order reversal curves beyond the Preisach model: An experimental permalloy microarray investigation
}

\author{
Felix Groß, ${ }^{*}$ Sven Erik Ilse, Gisela Schütz, Joachim Gräfe, ${ }^{\dagger}$ and Eberhard Goering ${ }^{\ddagger}$ \\ Max Planck Institute for Intelligent Systems, Stuttgart, Germany
}

(Received 11 June 2018; revised manuscript received 2 October 2018; published 1 February 2019)

\begin{abstract}
First-order reversal curves (FORCs) are a powerful tool to separate microscopic coercivities and interactions in a system without the need for lateral resolution. However, measured FORC densities are not always straightforward to interpret, especially if the system is interaction dominated and the Preisach-like interpretation of the FORC density breaks down. This is why FORC is often seen as a magnetic fingerprint instead of a measurement method yielding quantitative information. To understand additional features arising from the interactions in the system, we purposely designed permalloy microstructures which violate the Mayergoyz criteria. These artificial systems allow us to isolate the origin of an additional interaction peak in the FORC density. Modeling the system as a superposition of dipoles allows us to extract interaction strength parameters from this static simulation. Additionally, we suggest a linear relation between integrated interaction peak volume and interaction strength within the system. The presented correlation could be used to investigate the interaction behavior of samples as a function of structural parameters within a series of FORC measurements. This is an important step towards a more quantitative understanding of FORCs which violate the Mayergoyz criteria and away from a fingerprint interpretation.
\end{abstract}

DOI: 10.1103/PhysRevB.99.064401

\section{INTRODUCTION}

When investigating a magnetic system, its intrinsic properties are indispensable for a thorough characterization. Sophisticated measurement methods like magneto-optical Kerr microscopy give access to intrinsic magnetic properties such as magnetization states or coercive fields. However, these methods need resolutions in the order of magnitude of the observed feature. A more recent method is FORC (first-order reversal curve), which was developed by Mayergoyz in 1986 [1]. FORCs are a promising tool to get access to the Preisach distribution [2], a theoretical construct which includes information about all coercivities and interactions within a system. The major advantage of FORC is that it can yield microscopic information without the necessity for lateral resolution [3].

FORC has been successfully utilized to study an extensive amount of different magnetic systems. Whether for magnetic particles in geologic compounds [4-7], permanent magnet research [8-10], magnetic temperature dependence [11-13], the study of nanowires [14-18], or nanostructured dot and antidot systems [19-21], first-order reversal curves have a broad range of applications. It has been shown that FORC can also be extended to even more sophisticated methods like second-

\footnotetext{
*fgross@is.mpg.de

$\dagger$ graefe@is.mpg.de

${ }^{\ddagger}$ goering@is.mpg.de
}

order reversal curve (SORC) [22], or temperature FORC [23]. The amount of possible applications seems almost limitless.

Although FORC provides access to the interactions in a system [24-26] the corresponding FORC diagrams can become difficult to interpret as interaction features arise [27-29]. In a naive interpretation of FORC each peak corresponds to a specific component in the system of respective coercivity and interaction. However, as stated by Mayergoyz [1], this interpretation breaks down as soon as minor loops are not congruent or do not close after one field cycle. These two requirements are called wiping-out and congruency condition, respectively, of which the latter often does not hold for systems that feature interaction effects.

Dobrotă and Stancu [27] were able to explain how interactions affect FORC diagrams and how the so-called wishbone feature arises. This wishbone and other interaction features have been found and analyzed in several studies $[16,28,30-36]$. However, only a very small number of interaction features in FORC are well understood and even less are quantitatively interpretable.

This is why the FORC method itself is still not thoroughly understood, therefore sometimes referred to as a magnetic fingerprint [31,37], and to this day it is a topic of interest $[27,30,38]$. A more fundamental understanding of FORC and its complex behavior in the case of systems which violate the Mayergoyz criteria is necessary to reliably interpret FORC diagrams, to extract quantitative information from arising features, and to go beyond the magnetic fingerprint interpretation.

In order to better understand the FORC method itself artificially designed systems are well suited. To this end we studied different thin film structures based on the paper of Dobrotă and Stancu [27]. Targeted design allows for the creation of

Published by the American Physical Society under the terms of the Creative Commons Attribution 4.0 International license. Further distribution of this work must maintain attribution to the author(s) and the published article's title, journal citation, and DOI. 
structures that violate the Mayergoyz criteria. Knowing the composition of the structure, such as sample geometry and thickness and hence the stray field geometry, paves the way for the evaluation of the arising FORC densities. Revealing the origin of specific features within the FORC density would allow us to develop well-suited evaluation methods. This in turn would allow FORC to become a more quantitative measurement method even for non Preisach-like FORC densities, which might even extend the applicability of the method to systems with very complicated FORC diagrams.

\section{PREPARATION AND METHODS}

By using photolithography with direct laser writing it is possible to go from concept to sample within 1-2 days. Fast sample preparation is crucial to produce several systems with different structural parameters in order to then analyze a variety of different FORC densities.

To do so, we used photoresists by MicroChem and MicroChemicals, LOR 3A and AZ ECI 3027 respectively, and KLOÉ's Dilase 250 for the UV laser exposure.

All samples in this work consist of $50 \mathrm{~nm}$ thin permalloy and $2 \mathrm{~nm}$ aluminum as a capping layer for oxidization protection, grown on a silicon substrate. The thin film deposition was done with ion beam sputtering at pressures below $10^{-7}$ mbar. Having multiple samples on one substrate minimized both the time needed to switch samples between measurements and the difference in sputtering conditions during thin film deposition.

A FORC measurement can in principle be done with almost any device capable of measuring hysteresis curves [26]. For the FORC measurement itself the magnetization is not only measured as a function of the applied external $H$, but also depending on a so-called reversal field $H_{\mathrm{r}}$. Acquiring a first-order reversal curve starts at positive saturation. The applied field $H$ is then reduced to the first reversal field $H_{\mathrm{r}}$. The actual first-order reversal curve is then measured as magnetization from $H_{\mathrm{r}}$ back to positive saturation. This process is repeatedly executed with lower reversal fields until the reversal field hits negative saturation. The result is a twodimensional magnetization landscape $M\left(H, H_{\mathrm{r}}\right)$. The mixed second derivative

$$
\rho\left(H, H_{\mathrm{r}}\right) \equiv-\frac{1}{2} \frac{\partial^{2} M\left(H, H_{\mathrm{r}}\right)}{\partial H \partial H_{\mathrm{r}}}
$$

is the so-called FORC density, which yields information about the interaction and the coercivity distribution within the measured system. The following rotation and expansion of the coordinate system transforms the axis into a coercivity and an interaction axis, which allows for a more intuitive interpretation of the FORC diagram for simple systems:

$$
H_{\mathrm{u}}=\frac{1}{2}\left(H+H_{\mathrm{r}}\right), \quad H_{\mathrm{c}}=\frac{1}{2}\left(H-H_{\mathrm{r}}\right) .
$$

For a more extensive introduction to the FORC subject, the reader is referred to [27].

All measurements were carried out using Durham Magneto Optics' NANOMOKE3, which makes use of the magneto-optical Kerr effect (MOKE) [39,40] to measure magnetization curves. The most important point is the background correction when measuring FORCs with MOKE. Time- and field-dependent contributions have to be corrected in order to align the individual minor loops. This includes contributions such as field dependent Faraday effect and offset correction of the Kerr signal. The corresponding field sequence can be seen in Fig. 3 of reference [41]. For further information on how to set up a FORC measurement using MOKE, the reader is referred to elsewhere [41].

The MOKE FORC setup allows for the measurement of a complete set of FORC including 100 or more minor loops within 30-40 minutes. The resolution for the applied field can go down to $0.1 \mathrm{Oe}$. This allows one to complete FORC measurements much faster than in a conventional vibrating sample magnetometer, which is necessary to systematically modify several structural parameters.

Additionally, the MOKE was used to measure spatially resolved switching fields. To do so, the hysteresis of the structures is measured on approximately 250000 pixels on the sample with a resolution down to $1 \mu \mathrm{m}$ by taking pictures of the Kerr rotation while sweeping the major loop. This technique allows for both the measurement of hysteresis loops on each single element and a better understanding of the observed FORC densities. The measurement directly resolves switching fields of different regions of the sample.

In order to explain the arising interaction features, a static simulation was performed. In this simulation, the structures were approximated as multiple dipoles, one for each $\mu \mathrm{m}^{2}$. The magnetic field at the origin caused by dipole $i$ at position $\mathbf{r}_{i}$ can be written as [42]

$$
\mathbf{B}_{i}\left(\mathbf{r}_{i}\right)=-\frac{\mu_{0}}{4 \pi}\left(\frac{3 \mathbf{n}_{i}\left(\mathbf{n}_{i} \cdot \mathbf{m}\right)-\mathbf{m}}{\left|\mathbf{r}_{i}\right|^{3}}\right),
$$

where $\mathbf{n}$ is a unit vector along $\mathbf{r}, \mathbf{m}$ the magnetization vector, and $\mu_{0}$ the vacuum permeability.

The approximated interaction field of the sample at a given point $\mathbf{r}_{0}$ is then calculated as the sum of all dipole field contributions:

$$
\mathbf{B}_{\text {int }}\left(\mathbf{r}_{0}\right)=\sum_{i=1}^{n} \mathbf{B}_{i}\left(\mathbf{r}_{i}-\mathbf{r}_{0}\right) .
$$

The orientation of the dipoles can be changed by altering the direction of $\mathbf{m}$. In the simulation, this can be done separately for all individual parts of the structure, but only along the easy axis. By removing one part of the structure, the interaction field has been estimated by evaluating the field caused by all other dipoles in the sample.

\section{RESULTS}

\section{A. Building blocks: Single stripes}

In order to build systems violating the Mayergoyz criteria, pre-characterized building blocks are necessary. Using these building blocks, more complex systems will be designed later on.

The first sample consists of simple stripes and is displayed in Fig. 1(a). These permalloy stripes have different widths $(10,20, \ldots, 100 \mu \mathrm{m})$ and a length of $150 \mu \mathrm{m}$. Permalloy has a coercivity low enough to observe very small interaction effects in the FORC diagrams later on, such as magnetic stray fields from neighboring structures. The distance between 


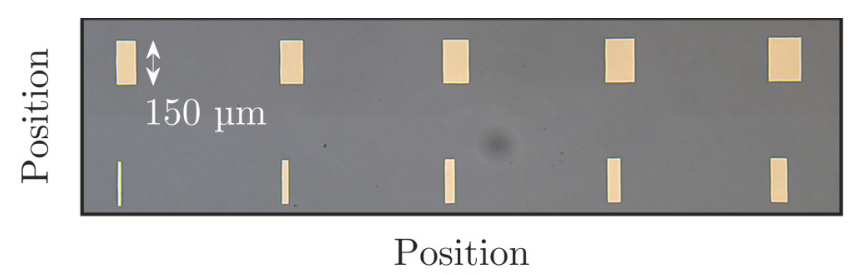

(a)

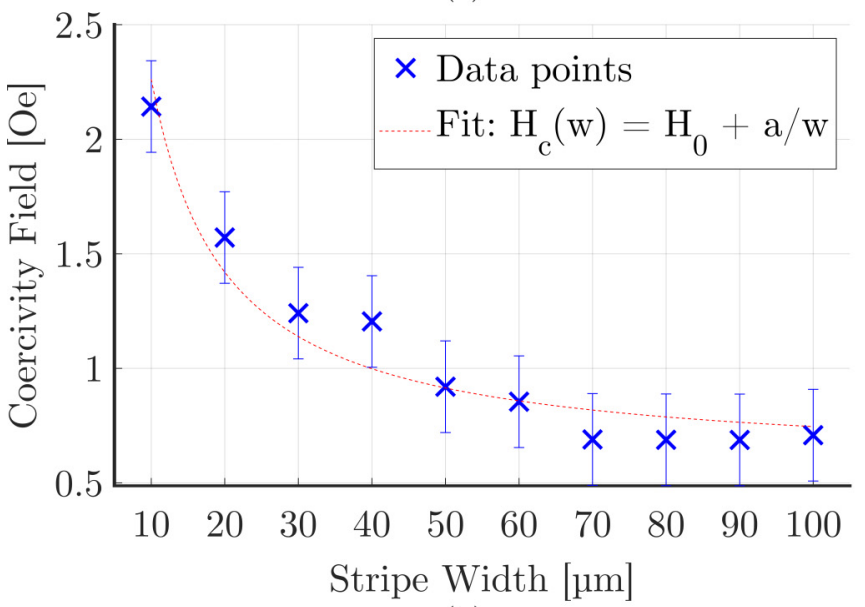

(b)

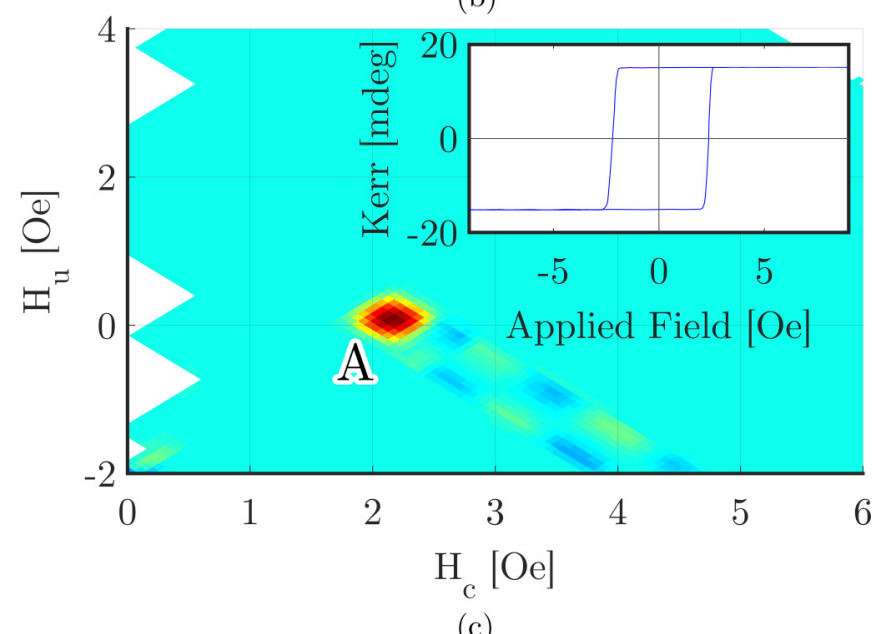

FIG. 1. Noninteraction stripes. (a) Microscope picture of isolated stripes (length $150 \mu \mathrm{m}$, width $10-100 \mu \mathrm{m}$. (b) Coercivity as a function of stripe width. (c) FORC density (base line resolution after smoothing $=1$ Oe [41]) and major loop of smallest stripe $(10 \mu \mathrm{m})$.

the stripes in this first model system is so large that their interaction can be neglected (noninteracting case). This allows for the measurement of the unperturbed hysteresis of these single stripes as well as the calculation of their coercivity.

The external field is always aligned along the long axis of the permalloy stripe. Since this is the shape anisotropy related easy axis, each stripe shows a typical square shaped hysteresis loop [shown in the inset of Fig. 1(c)] with no static intermediate state.

The measured coercivity is plotted in Fig. 1(b) as a function of stripe width. It has been shown $[43,44]$ that the coercivity of such a system scales with the inverse width. The smallest stripe has a coercivity of about 2.2 Oe. For medium widths
$(50 \mu \mathrm{m})$, the coercivity is about $1 \mathrm{Oe}$ and approaches $0.7 \mathrm{Oe}$ for the widest stripes.

When measuring a set of FORCs on the most narrow stripe, one finds exactly the expected FORC diagram [Fig. 1(c)]. There is one peak (peak A) in the FORC density, located at $H_{\mathrm{u}}=0$ (no interaction) and $H_{\mathrm{c}}=2.2 \mathrm{Oe}$, which corresponds to the coercivity of the major loop.

\section{B. Complex systems: Alternating stripes}

Arranging eleven basic stripes of alternating width into one interacting structure creates a more complex system which violates the Mayergoyz criteria. The structure of such a system is shown in Fig. 2(a).

For these measurements, the laser spot needs to be slightly defocused to cover the entire area of the structure. The full width at half maximum of the laser spot should be larger than the structure so as to resolve all structural features. The size of the illuminated area should be balanced out with respect to the signal-to-noise ratio of the magnetization curves.

The corresponding FORC density, measured along the long axis of the stripes, is shown in Fig. 2(b). For a better illustration of the switching field distribution below, the density is displayed in $H$ and $H_{\mathrm{r}}$ coordinates. Judging from the FORC density before, one would naively expect two peaks, one for each coercivity component (stripe width). However, four peaks are visible, three positive and one negative. If the system was completely free of interaction, only peaks A and B would be visible in the FORC density: these are the two peaks corresponding to the switching of the two different stripe widths. However, there is a positive-negative peak pair (peak pair C), which does not correspond to a specific component within the sample.

In contrast, the switching field or susceptibility $\chi(H)$ (FORC density integrated in the $H_{\mathrm{r}}$ direction, equal to the first derivative of the major loop) only shows two peaks. Those correspond to the switching events of the two different stripe widths. There is, however, no additional peak to be found here.

A completely independent measure of the switching field is presented in Fig. 2(c). This is the spatially resolved switching field, the acquisition of which is described in the method section.

The color map displays the local switching field as a function of position. When comparing it to the sketch of the sample on the illustration above, it can be seen that the two coercivities of the two different stripe widths are well separated. Small stripes are displayed in orange-red, big stripes in blue. The blue color represents a relatively low switching field ( $\sim 1 \mathrm{Oe})$, the red color represents a comparatively high switching field ( $\sim 3 \mathrm{Oe})$. As shown before in Fig. 2(d), we cannot see a switching field component for each peak in the FORC diagram. These two components only explain the two peaks at $H_{\mathrm{u}}=0$.

The small darker parts at the corners of the big stripes are nucleation points for the domain wall. These are the points where domain walls start nucleating. However, the other parts of the stripes are rather homogeneous, which shows that each stripe flips entirely once a domain wall nucleates. It seems that domain wall pinning in these soft magnetic permalloy stripes is much weaker than the applied critical field. 


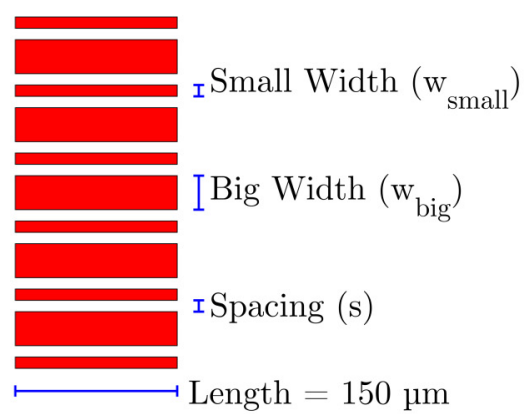

(a)

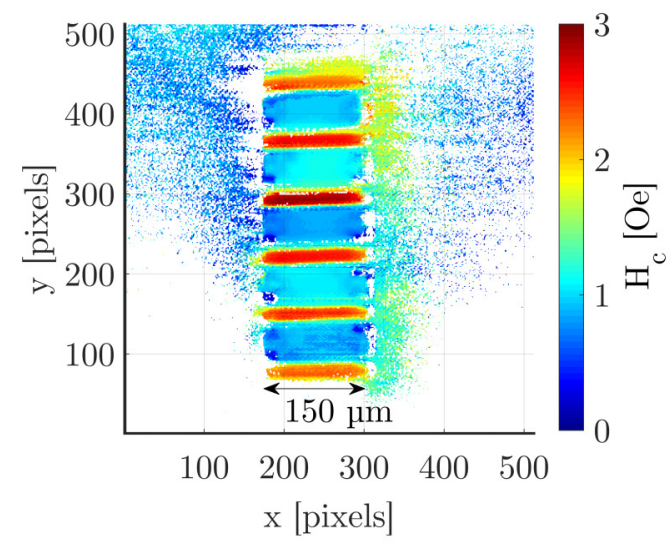

(c)

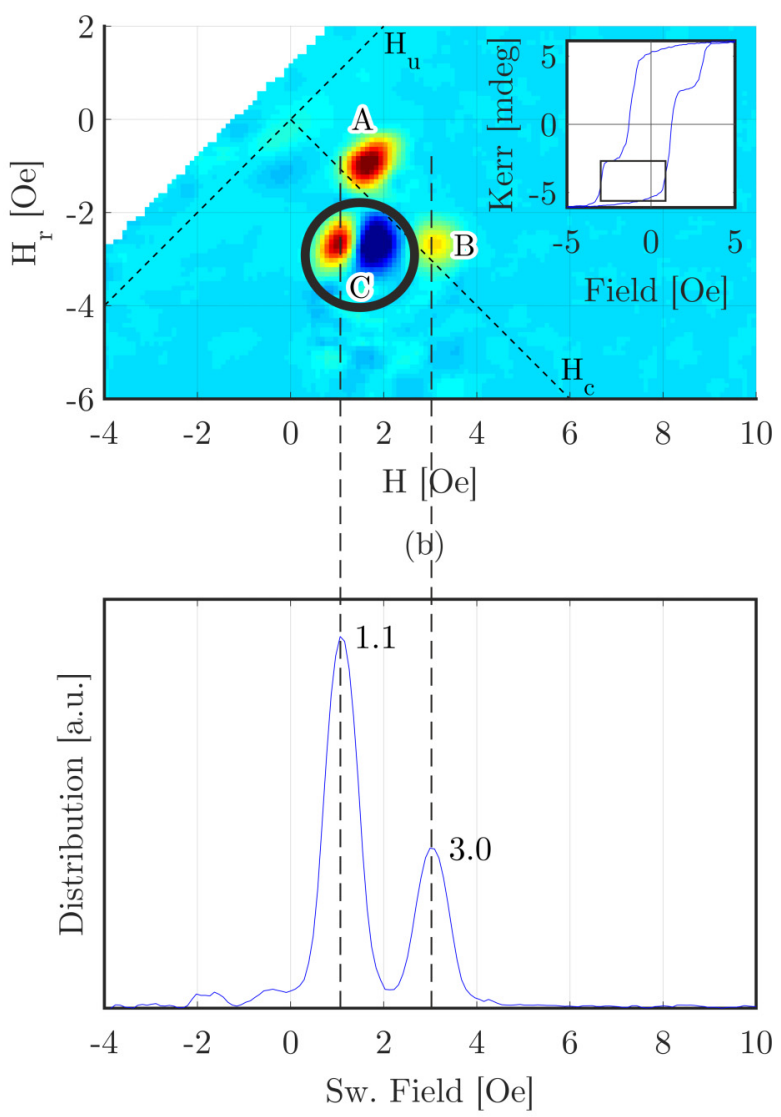

(d)

FIG. 2. Alternating stripes: (a) Schematic structure (small width $=10 \mu \mathrm{m}$, big width $=30 \mu \mathrm{m}$, spacing $=10 \mu \mathrm{m}$ ). (b) FORC density (baseline resolution after smoothing $=1$ Oe [41]) and major loop of the structure; note that the FORC density is presented in $H$ and $H_{\mathrm{r}}$ coordinates so that the $H$ coordinate matches the switching field. The projection in the $H_{\mathrm{r}}$ direction is the switching field distribution shown in (d). (c) Spatially resolved switching field of the structure. (d) switching field distribution calculated as the projection of the FORC density in the $H_{\mathrm{r}}$ direction.

It is clear that that the explanation of the additional peak pair is not as straightforward as the explanation for peaks A and B. Apparently, the peak pair cannot be explained using the simple Preisach-like interpretation approach. So what exactly is the origin of these peaks?

The beginning of the explanation lies in the different magnetization configurations (Fig. 3) in which switching events appear in this particular structure. The illustration shows the magnetization configurations of the major loop depending on the applied field. The color code in the major loop shows the respective configuration. The arrows denote the direction in which the magnetization of the respective stripe is pointing. Starting a minor loop in negative saturation (yellow state) and driving up the external field towards positive saturation (green) leads to two different switching events. The first one is the switching of the big stripes from parallel configuration (yellow) into antiparallel configuration (red). This switching event A corresponds to peak A in the FORC density in Fig. 2(b). Going further towards positive saturation will make the small stripes flip from antiparallel (red) back into parallel geometry (green, event B) where saturation is reached.

However, there is a second minor loop, which also has a strong impact onto the FORC density. This minor loop starts in antiparallel geometry (blue) when the sample has not been fully saturated in the negative direction. A sketch of the minor loop starting in the blue configuration is shown as a solid blue line in the plot. This minor loop also corresponds to the switching of the big stripes, like A. However, in this case the switching happens in antiparallel geometry (event C), unlike before. As a consequence, the interaction, which supported the switching event before, hinders it this time. Hence, the switching event $\mathrm{C}$ occurs at higher external fields than event A. Had there been no interaction, both switching events would have happened at the same applied field (illustrated by the dashed blue line) and the minor loops would have been congruent within their overlapping field ranges. In other words, the interaction between the lower and higher coercivity components causes the big stripes to flip at different external fields depending on the magnetization geometry. This, however, violates the Mayergoyz congruency property, which then generates additional features (peak pair C) in the FORC density.

The interaction shift, denoted as a red arrow in the inset, is linearly related to the interaction strength of the system, which will be important later when quantifying the interaction feature in the FORC density.

From the $H_{\mathrm{u}}$ and $H_{\mathrm{c}}$ coordinates of the resulting peak we can calculate the corresponding reversal and applied field. We 


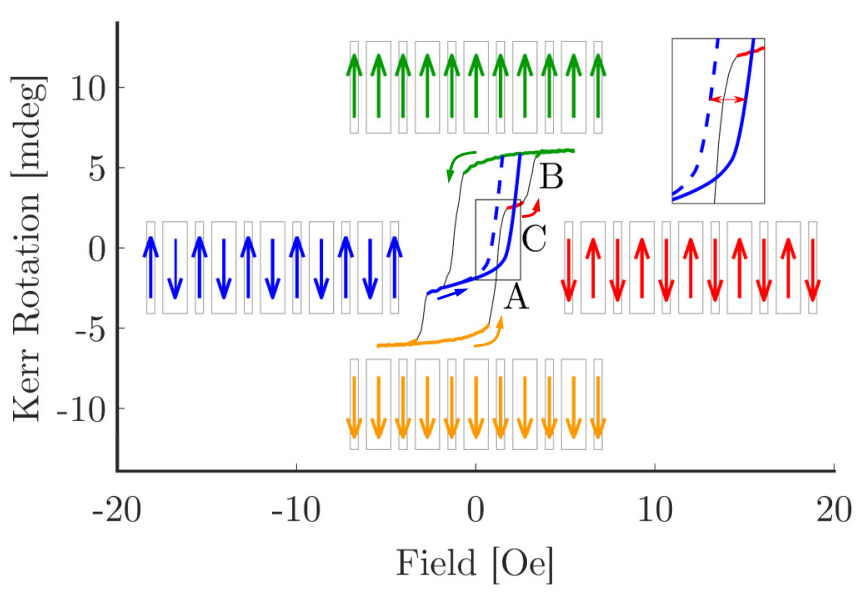

FIG. 3. Illustration of the different magnetization configurations at four different positions of the major loop. The inset displays the exaggerated minor loop shift caused by the interaction. The capital letters denote switching events: A and B are the two main switching events within the major loop, $\mathrm{C}$ denotes the switching event of the minor loop.

find that this reversal and applied field exactly correspond to the lower left part of the major loop [see inset, Fig. 2(b)]. Hence, the position of the interaction peak in the FORC diagram can then be calculated as

$$
H_{\mathrm{u}}=\frac{1}{2}\left(H_{\mathrm{c}, \text { low }}-H_{\mathrm{c}, \text { high }}\right)
$$

and

$$
H_{\mathrm{c}}=\frac{1}{2}\left(H_{\mathrm{c}, \text { low }}+H_{\mathrm{c}, \text { high }}\right),
$$

where $H_{\mathrm{c}, \text { low }}$ and $H_{\mathrm{c}, \text { high }}$ denote the $H_{\mathrm{c}}$ coordinate of the low and the high coercivity peak respectively. This property could be used to isolate interaction features from features which correspond to actual components of the sample.

\section{Structural parameter variation}

In order to attain systematic variations of coupling strength and coercivity parameters, several samples have been designed. The varied parameters were the width ( $w_{\text {big }}$ ) of the big stripe (coercivity) and the spacing $(s)$ in between the stripes (coupling). For each of the big stripe widths of 30 and $50 \mu \mathrm{m}$ there are three different spacings of 10,20 , and $30 \mu \mathrm{m}$, which adds up to a total of six different samples.

The measured FORCs on these six samples can be seen in Fig. 4. Note that they are presented in $H_{\mathrm{c}}$ and $H_{\mathrm{u}}$ coordinates this time. All of them show the same three prominent features presented before: A low coercivity peak A, a high coercivity peak $\mathrm{B}$, and an interaction peak pair $\mathrm{C}$ at negative $H_{\mathrm{u}}$. The additional insets in each diagram display the major loops.

When comparing the $H_{\mathrm{c}}$ position of the high coercivity peak B of sample $w_{\text {big }}=30 \mu \mathrm{m}, s=10 \mu \mathrm{m}$ (top left) with sample $w_{\text {big }}=30 \mu \mathrm{m}, s=30 \mu \mathrm{m}$ (top right), the peak visibly shifts to lower coercivities with increasing spacing. In a naive interpretation approach, that should not be the case. The coercivity of the small stripe (high coercivity) of constant width $(10 \mu \mathrm{m})$ should not decrease when the spacing between the stripes is increased. However, the reason for this can be found in the method itself [27]: The highest coercivity component of a sample (small stripes in this case) will always flip last. The reversal field $H_{\mathrm{r}}$, at which the sample switches down, is the intrinsic coercivity plus interaction, $H_{\mathrm{r}}=-\left(H_{\text {intrinsic }}+H_{\text {interaction }}\right)$. The applied field $H$, at which the sample switches back up, is also its intrinsic coercivity plus interaction, $H=H_{\text {intrinsic }}+H_{\text {interaction }}$, except without the minus. If we use formula 2 to transform this into $H_{\mathrm{u}}$ and $H_{\mathrm{c}}$, we see that

$$
\begin{aligned}
H_{\mathrm{u}} & =\frac{1}{2}\left(H+H_{\mathrm{r}}\right) \\
& =\frac{1}{2}\left[H_{\text {intrinsic }}+H_{\text {interaction }}-\left(H_{\text {intrinsic }}+H_{\text {interaction }}\right)\right] \\
& =0
\end{aligned}
$$

and

$$
\begin{aligned}
H_{\mathrm{c}} & =\frac{1}{2}\left(H-H_{\mathrm{r}}\right) \\
& =\frac{1}{2}\left(H_{\text {intrinsic }}+H_{\text {interaction }}+H_{\text {intrinsic }}+H_{\text {interaction }}\right) \\
& =H_{\text {intrinsic }}+H_{\text {interaction }} .
\end{aligned}
$$

This means that $H_{\mathrm{u}}$ is necessarily 0 if the major loop is symmetric and that the interaction is instead added onto the coercivity axis as a coercivity shift $H_{\text {interaction [27]. }}$.

To obtain a quantitative measure for the coercivity shift, simulations were performed using a static dipole approximation described in the method section. By running the simulation with all dipoles of one stripe removed, the interaction resulting on that stripe can be estimated. Furthermore, the model is capable of switching individual stripes by switching the direction of all dipoles of the corresponding stripe.

To measure the interaction field acting on one particular stripe, the resulting field at the center position of each stripe was taken while that stripe was removed. However, the domain wall nucleation and therefore the flipping process predominantly starts at the short end of a stripe since the field is applied along its long axis. In the simulation, when going from the center to the short end of a stripe, the field can differ dramatically: by about two orders of magnitude, and it can even go down to 0 . However, the interaction at the center is still a good relative measure for the interaction, although of different magnitude. Eventually, a relative measure is still sufficient for the analysis conducted here and is a great success considering the simplicity of the model.

In order to account for the difference between simulation and the real nucleation process at the short end of a stripe, we scaled the interaction values of the model down by a constant factor $(\approx 20)$ so that the modeled data in Fig. 5 fit the experiment. This is valid since the values from this model are only taken as relative values representing the experiment.

The results of this model and the real shift of coercivity of the small stripes are demonstrated in Fig. 5. In the following, four different trends are plotted as a function of stripe spacing (interaction strength). Blue plots illustrate the coercivity shift calculated as the difference between the $H_{\mathrm{c}}$ coordinate of the corresponding peak from the FORC diagrams and the coercivity of the isolated stripe $(2.2 \mathrm{Oe})$. The red plots illustrate the 

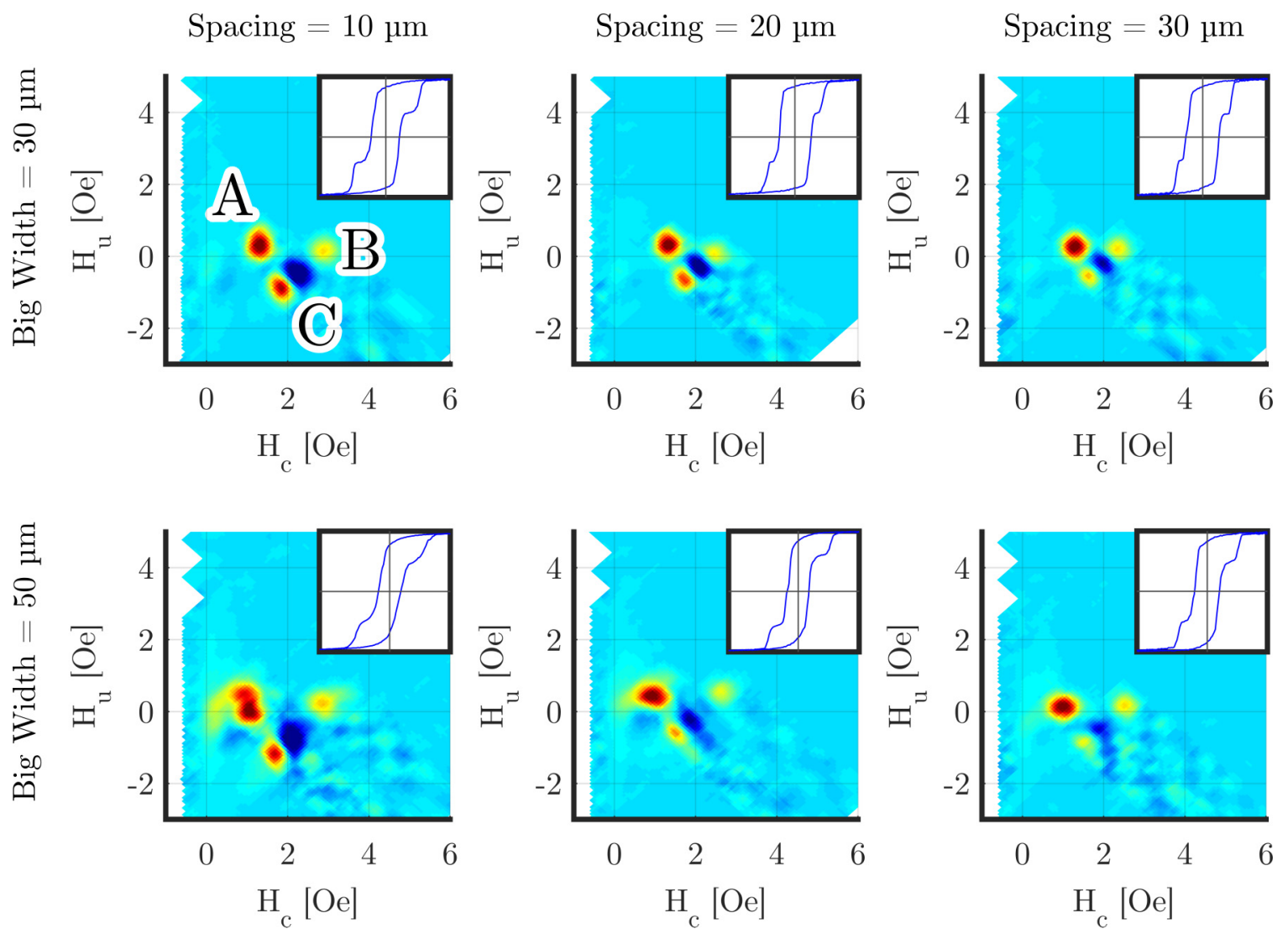

FIG. 4. FORC densities of six different samples with systematically varied structural parameters. This time the FORC densities are displayed in the usual $H_{\mathrm{u}}, H_{\mathrm{c}}$ coordinates. Insets display the shapes of the major loops.

coercivity shift extracted from the dipole model in arbitrary units. Triangles denote big stripe widths of $w_{\text {big }}=30 \mu \mathrm{m}$, squares denote $w_{\text {big }}=50 \mu \mathrm{m}$. The dashed lines are included to better illustrate the descending trend of the four different curves.

When comparing the simulation with the measurement, the coercivity shift seems to saturate in all four cases when going to higher spacings. This is reasonable as the coercivity shift should approach 0 for high spacings when the stripes are not

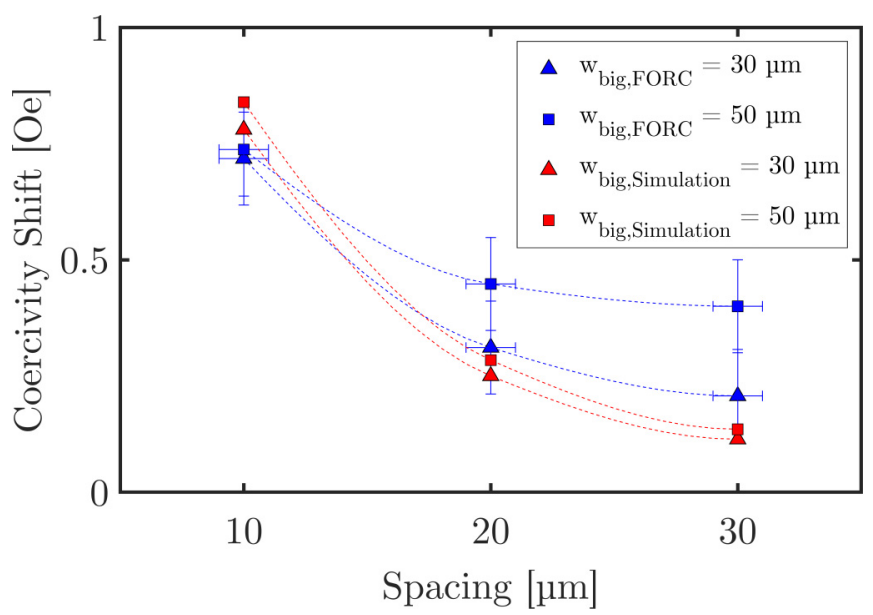

FIG. 5. Comparison between the coercivity shift measured in FORC and the estimated coercivity shift from the dipole model. interacting anymore. Additionally, in the experiment as well as in the simulation the shift is higher for $w_{\text {big }}=50 \mu \mathrm{m}$, which also makes sense since the stray field acting on the small stripes should be higher for wider stripes in between. In general, the model seems to be able to reproduce the trend of the measurement. It certainly has its limitations and there are some discrepancies with the experiment, which, however, is to be expected for a rough dipole approximation.

When looking at the FORC diagram, the coercivity shift is not the only changing feature when varying the structural parameters. The intensity of the interaction peak pair decreases for both widths when the spacing between the stripes is increased (going from left to right). This is a result of the decrease in coupling when the spacing between the stripes is increased. The interaction shift (denoted by the red double arrow in the inset of Fig. 3) decreases when the coupling strength is decreased (spacing is increased). If the spacing reaches a distance at which coupling is not relevant anymore, the interaction shift approaches 0 and the stripes are completely uncoupled. In this case, the interaction peak would completely vanish. This already implies a relation between the interaction peak intensity and the interaction field acting on the big stripes.

The integrated interaction peak volume has the same dimension as the integrated FORC density [45], and therefore corresponds to a magnetization. In this case, the magnetization contained in the interaction peak approximately corresponds to the "incongruency" of the two respective minor 


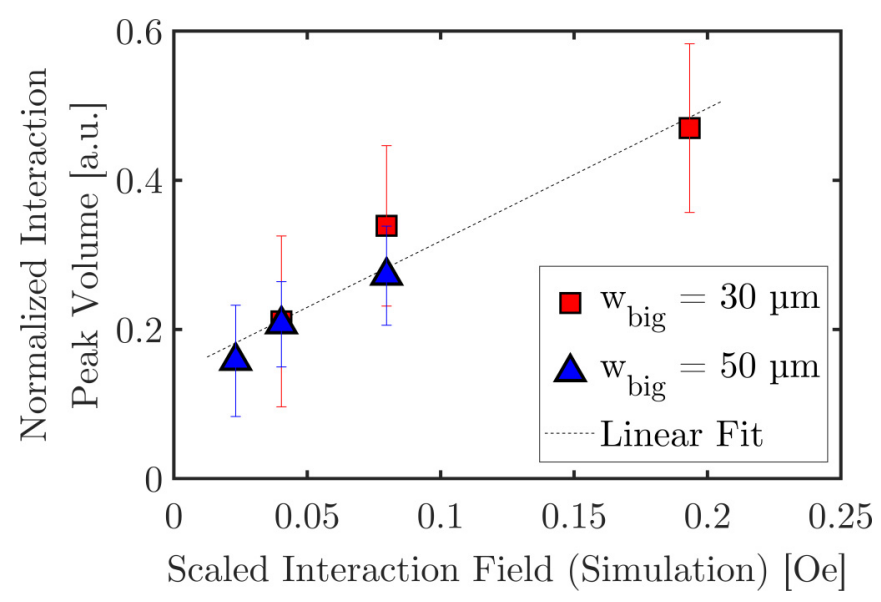

FIG. 6. Normalized interaction peak volume plotted as a function of simulated interaction strength acting on the big stripes. The field extracted from the simulation was scaled by the same factor as before.

loops within their overlapping field ranges (the one starting at negative saturation and the one sketched by the solid blue line in Fig. 3).

There are two factors influencing the size of the interaction peak. The first is the difference in slope between the two minor loops, and the second is the field range in which these slopes are different. The first one is not affected by the configuration of the sample; the latter one is. Differences in field range exactly correspond to the interaction shift (Fig. 3) for small interactions. Hence, we suggest a linear relation between the integrated interaction peak volume and the interaction which shifts the switching process of the big stripes in the corresponding minor loops.

In Fig. 6, the normalized interaction peak volume is plotted against the scaled interaction field acting on the big stripes, which was extracted from the simulation. The interaction from the simulation is used as a measure of the interaction shift of the minor loop. The peak volume is normalized with respect to the saturation Kerr angle. Both quantities are a measure of the interaction in the system. Therefore, plotting them against each other yields the relation between interaction peak volume and real interaction in the system.

As suggested before, we introduced a linear relation for the interaction peak volume as a function of the real interaction strength, which should be valid for the assumption of small interaction fields. Both widths $\left(w_{\text {big }}=30,50 \mu \mathrm{m}\right)$ are considered equally for the fit since the higher stray field yields higher interaction peak volumes but equally increases the stray field in the simulation. This cancels out if they are plotted against each other. This linear relation can be a first model to demonstrate the connection between the interaction strength and the peak volume, which seems to be valid within the range which is accessible with our samples.

The interaction peak volume is not the only interesting quantity. The position of both interaction peaks with respect to each other can also yield information about the type of interaction. In this case, all of the structures are coupled antiparallelly due to their stray fields. This means that, in saturation, the flipping of the low coercivity component is favored by the field geometry. In contrast, the flipping from antiparallel geometry back to saturation is not favored by the stray field. Thus, the solid blue line in Fig. 3 crosses the major loop and the interaction peak pair appears first positive then negative when going from negative to positive $H$ values in Fig. 2(b).

If the interaction was the other way round-with a stray field that favors parallel alignment, and the solid blue line shifted towards smaller external fields than the major loopthe interaction peak would appear the other way round, that is first negative then positive when going from negative to positive $H$ values.

In this simple case, the interaction peak volume is a measure of the interaction within the system. This can be used, for example, to compare interactions in nonartificial systems as a functions of different parameters. This model needs to be further quantified, especially for different samples. Perhaps the linear relation is a good first approximation but can be further adapted for other ranges of interaction and different sets of samples. In principle, it would be possible to find the exact relation between interaction peak volume and interaction strength for a specific material, which would allow for it to be used as a measure of the interaction between soft and hard magnetic phases within.

\section{DISCUSSION}

We studied the behavior of FORC in the presence of interactions based on the paper of Dobrotă and Stancu [27]. We found similar additional features; however, their interpretation is very complex if the exact composition and field geometry of the sample is unknown. This is where our artificial system can stand out.

The artificially created composition of binary coercivities and the perfect knowledge about the system allow us to easily assign the different peaks to the respective parts of the sample.

In the insets of Fig. 4, the area of the major loop between the two switching processes A and B hardly shows any magnetization change. Thus, the peaks corresponding to different coercivities in the FORCs are relatively well separated. This is the binary limit of the wishbone-like shape presented by Dobrotă and Stancu [27], whereas the wishbone is the limit of continuous coercivity and interaction field distributions. In the binary limit, the interaction peak volume can be easily determined. This is not self-evident since interaction features often merge with main coercivity peaks if the coercivity distribution is continuous.

With that in mind, the applicability of the presented model can become challenging. If the real coercivity peak is not easily separable from the interaction features, the peak volume cannot be determined precisely and therefore the model does not hold true. However, this model should be applicable if there are two phases that are well separated in switching field.

In this case, the volume determination can easily be done, and one is able to draw conclusions regarding the interaction strength in the system, even if the interaction is so small that there is hardly any shift of the low coercivity peak in the $H_{\mathrm{u}}$ direction and the wishbone shape is not present. 


\section{SUMMARY}

Artificially designed systems of $50 \mathrm{~nm}$ thick permalloy stripes have been investigated using MOKE-FORC [41]. When investigating single isolated stripes of different width, we find the expected coercivity scaling $[43,44]$ and the FORC peak corresponding to the coercivity of the stripe. Using these simple objects to set up more complicated geometries of alternating stripes, we find that the resulting FORC diagram shows a double peak feature, which can be ascribed to the interaction within the system [29]. Furthermore, the high coercivity peak shifts to higher values due to its interaction with the low coercivity component [27]. Both of these features are a consequence of the stray field geometry and the resulting interaction in the sample.

In order to evaluate these features, we developed a model which treats each of the stripes in the system as a superposition of dipoles. The resulting field of all dipoles is a measure of the interaction field in the system at a given point of the sample. By deactivating one of the stripes in the simulation, we can extract the interaction field acting on that particular stripe in arbitrary units. By comparing the interaction fields of different sample geometries, we can extract a trend from the simulation and compare it to the coercivity shift of the real sample. The model is not able to perfectly resolve all features; however, it is able to predict trends when comparing different sample geometries.

Exactly knowing our sample enabled us to identify the origin of the interaction peak pair feature as a shift of the minor loop starting in antiparallel geometry with respect to the minor loop starting in negative saturation. This shift is caused by the stray field of the small stripes, which shifts the switching field of the big stripes depending on the magnetization state. In order to obtain a quantitative measure for this interaction, we evaluated the volume of the positive interaction peak and compared it to the interaction predicted by the dipole model. For the range covered by our samples, we suggest a linear behavior for these two quantities.

The approach of comparing different interaction peak volumes seems promising to compare interaction strengths in similar samples with systematic parameter variation, for example as a function of alloying levels or temperature. This could come in handy if the interaction effects are rather small and the $H_{\mathrm{u}}$ shift is thus easy to miss.

FORC data interpretation can be difficult, especially if interaction features arise and the FORC density differs from the easily interpretable Preisach distribution. We suggest that features which arise in the FORC density should be seen as an additional source of information about the system instead of a troublesome problem which limits the interpretability. By identifying the fundamental origin of these features, it is possible to develop additional ways of extracting information from FORC densities. This interaction peak analysis is a thorough step for FORC to become a more quantitative measurement method instead of relying on the fingerprint interpretation.

\section{ACKNOWLEDGMENT}

The authors thankfully acknowledge Bernd Ludescher for thin film deposition.
[1] I. D. Mayergoyz, Mathematical Models of Hysteresis, Phys. Rev. Lett. 56, 1518 (1986).

[2] F. Preisach, Über die magnetische Nachwirkung, Z. Phys. A 94, 277 (1935).

[3] F. Béron, L.-P. Carignan, D. Ménard, and A. Yelon, Extracting individual properties from global behaviour: First-order reversal curve method applied to magnetic nanowire arrays, in Electrodeposited Nanowires and Their Applications, edited by $\mathrm{N}$. Lupu (IntechOpen, London, 2010), Chap. 7.

[4] A. P. Roberts, C. R. Pike, and K. L. Verosub, First-order reversal curve diagrams: A new tool for characterizing the magnetic properties of natural samples, J. Geophys. Res. B 105, 28461 (2000).

[5] A. P. Chen, R. Egli, and B. M. Moskowitz, First-order reversal curve (FORC) diagrams of natural and cultured biogenic magnetic particles, J. Geophys. Res. 112, (2007).

[6] A. R. Muxworthy and A. P. Roberts, First-order reversal curve (FORC) diagrams, in Encyclopedia of Geomagnetism and Paleomagnetism (Springer-Verlag, Berlin, 2007), p. 266.

[7] T. Yamazaki, Environmental magnetism of Pleistocene sediments in the North Pacific and Ontong-Java Plateau: Temporal variations of detrital and biogenic components, Geochem. Geophys. 10, (2009).

[8] H. Chiriac, N. Lupu, L. Stoleriu, P. Postolache, and A. Stancu, Experimental and micromagnetic first-order reversal curves analysis in NdFeB-based bulk "exchange spring"-type permanent magnets, J. Magn. Magn. Mater. 316, 177 (2007).
[9] T. Schrefl, T. Shoji, M. Winklhofer, H. Oezelt, M. Yano, and G. Zimanyi, First order reversal curve studies of permanent magnets, J. Appl. Phys. 111, 07A728 (2012).

[10] P.-A. Chen, C.-Y. Yang, S.-J. Chang, M.-H. Lee, N.-K. Tang, S.-C. Yen, and Y.-C. Tseng, Soft and hard natures of $\mathrm{Nd}_{2} \mathrm{Fe}_{14} \mathrm{~B}$ permanent magnet explored by first-order-reversal-curves, J. Magn. Magn. Mater. 370, 45 (2014).

[11] S. Muralidhar, J. Gräfe, Y.-C. Chen, M. Etter, G. Gregori, S. Ener, S. Sawatzki, K. Hono, O. Gutfleisch, H. Kronmüller, G. Schütz, and E. J. Goering, Temperature-dependent firstorder reversal curve measurements on unusually hard magnetic low-temperature phase of MnBi, Phys. Rev. B 95, 024413 (2017).

[12] T. Yomogita et al., Temperature and field direction dependences of first-order reversal curve (FORC) diagrams of hot-deformed Nd-Fe-B magnets, J. Magn. Magn. Mater. 447, 110 (2018).

[13] M. Rivas, J. C. Martínez-García, and P. Gorria, Visualizing decoupling in nanocrystalline alloys: A FORC-temperature analysis, J. Magn. Magn. Mater. 400, 315 (2016).

[14] F. Béron, L. Clime, M. Ciureanu, D. Menard, R. W. Cochrane, and A. Yelon, First-order reversal curves diagrams of ferromagnetic soft nanowire arrays, IEEE Trans. Magn. 42, 3060 (2006).

[15] F. Béron, L. P. Carignan, D. Menard, and A. Yelon, Magnetic behavior of $\mathrm{Ni} / \mathrm{Cu}$ multilayer nanowire arrays studied by firstorder reversal curve diagrams, IEEE Trans. Magn. 44, 2745 (2008). 
[16] F. Béron, L. Clime, M. Ciureanu, D. Ménard, R. W. Cochrane, and A. Yelon, Magnetostatic interactions and coercivities of ferromagnetic soft nanowires in uniform length arrays, J. Nanosci. Nanotechnol. 8, 2944 (2008).

[17] C.-I. Dobrotă and A. Stancu, Tracking the individual magnetic wires' switchings in ferromagnetic nanowire arrays using the first-order reversal curves (FORC) diagram method, Physica B (Amsterdam) 457, 280 (2015).

[18] M. Ciureanu, F. Béron, P. Ciureanu, R. W. Cochrane, D. Ménard, A. Sklyuyev, and A. Yelon, First order reversal curves (FORC) diagrams of Co nanowire arrays, J. Nanosci. Nanotechnol. 8, 5725 (2008).

[19] C. Pike and A. Fernandez, An investigation of magnetic reversal in submicron-scale Co dots using first order reversal curve diagrams, J. Appl. Phys. 85, 6668 (1999).

[20] J. Gräfe, M. Weigand, C. Stahl, N. Träger, M. Kopp, G. Schütz, E. J. Goering, F. Haering, P. Ziemann, and U. Wiedwald, Combined first-order reversal curve and $\mathrm{x}$-ray microscopy investigation of magnetization reversal mechanisms in hexagonal antidot lattices, Phys. Rev. B 93, 014406 (2016).

[21] J. Gräfe, M. Weigand, N. Träger, G. Schütz, E. J. Goering, M. Skripnik, U. Nowak, F. Haering, P. Ziemann, and U. Wiedwald, Geometric control of the magnetization reversal in antidot lattices with perpendicular magnetic anisotropy, Phys. Rev. B 93, 104421 (2016).

[22] A. Stancu, P. Andrei, and L. Stoleriu, Magnetic characterization of samples using first- and second-order reversal curve diagrams, J. Appl. Phys. 99, 08D702 (2006).

[23] V. Franco, T. Gottschall, K. P. Skokov, and O. Gutfleisch, First-order reversal curve (FORC) analysis of magnetocaloric Heusler-type alloys, IEEE Magn. Lett. 7, 6602904 (2016).

[24] C. R. Pike, A. P. Roberts, and K. L. Verosub, Characterizing interactions in fine magnetic particle systems using first order reversal curves, J. Appl. Phys. 85, 6660 (1999).

[25] M. Nica and A. Stancu, FORC diagram study of magnetostatic interactions in 2D longitudinal arrays of magnetic wires, Physica B (Amsterdam) 475, 73 (2015).

[26] M. Rivas, P. Gorria, C. Munoz-Gomez, and J. C. MartinezGarcia, Quasi-static AC FORC measurements for soft magnetic materials and their differential interpretation, IEEE Trans. Magn. 53, 2003606 (2017).

[27] C.-I. Dobrotă and A. Stancu, What does a first-order reversal curve diagram really mean? A study case: Array of ferromagnetic nanowires, J. Appl. Phys. 113, 043928 (2013).

[28] C. R. Pike, C. A. Ross, R. T. Scalettar, and G. T. Zimanyi, Firstorder reversal curve diagram analysis of a perpendicular nickel nanopillar array, Phys. Rev. B 71, 134407 (2005).

[29] V. Franco, F. Béron, K. R. Pirota, M. Knobel, and M. A. Willard, Characterization of the magnetic interactions of multiphase magnetocaloric materials using first-order reversal curve analysis, J. Appl. Phys. 117, 17C124 (2015).
[30] D. A. Gilbert, G. T. Zimanyi, R. K. Dumas, M. Winklhofer, A. Gomez, N. Eibagi, J. L. Vicent, and K. Liu, Quantitative decoding of interactions in tunable nanomagnet arrays using first order reversal curves, Sci. Rep. 4, 4204 (2014).

[31] B. F. Valcu, D. A. Gilbert, and K. Liu, Fingerprinting inhomogeneities in recording media using the first-order reversal curve method, IEEE Trans. Magn. 47, 2988 (2011).

[32] E. Jafari-Khamse, M. Almasi Kashi, and A. Ramazani, Firstorder-reversal-curve (FORC) diagrams of alternative chain of soft/hard magnetic $\mathrm{CoFe} / \mathrm{Cu}$ multilayer nanowires, Curr. Appl. Phys. 16, 486 (2016).

[33] J. García, V. M. Prida, V. Vega, W. O. Rosa, R. Caballero-Flores, L. Iglesias, and B. Hernando, 2D and 3D ordered arrays of Co magnetic nanowires, J. Magn. Magn. Mater. 383, 88 (2015).

[34] J. García, V. M. Prida, L. G. Vivas, B. Hernando, E. D. Barriga-Castro, R. Mendoza-Reséndez, C. Luna, J. Escrig, and M. Vázquez, Magnetization reversal dependence on effective magnetic anisotropy in electroplated $\mathrm{Co}-\mathrm{Cu}$ nanowire arrays, J. Mater. Chem. C 3, 4688 (2015).

[35] F. Béron, L. A. S. de Oliveira, M. Knobel, and K. R. Pirota, A novel method for identifying the local magnetic viscosity process of heterogeneous magnetic nanostructures, J. Phys. D 46, 045003 (2013).

[36] E. P. Vetter, P. Ghatwai, W. A. Soffa, and J. A. Floro, Evolution of first-order reversal curves during self-assembly of the $\mathrm{Co}_{40.2} \mathrm{Pt}_{59.8}$ nano-chessboard structure, IEEE Magn. Lett. 6, 6600104 (2015).

[37] R. K. Dumas, C.-P. Li, I. V. Roshchin, I. K. Schuller, and K. Liu, Magnetic fingerprints of sub-100 nm Fe dots, Phys. Rev. B 75, 134405 (2007).

[38] F. Béron, L. Clime, M. Ciureanu, D. Ménard, R. W. Cochrane, and A. Yelon, Reversible and quasireversible information in first-order reversal curve diagrams, J. Appl. Phys. 101, 09J107 (2007).

[39] J. Kerr, XLIII. On rotation of the plane of polarization by reflection from the pole of a magnet, Philos. Mag. 3, 321 (1877).

[40] J. Kerr, XXIV. On reflection of polarized light from the equatorial surface of a magnet, Philos. Mag. 5, 161 (1878).

[41] J. Gräfe, M. Schmidt, P. Audehm, G. Schutz, and E. Goering, Application of magneto-optical Kerr effect to first-order reversal curve measurements, Rev. Sci. Instrum. 85, 023901 (2014).

[42] J. D. Jackson, Classical Electrodynamics, 3rd ed. (John Wiley \& Sons, New York, 1999).

[43] J. Gräfe, G. Schütz, and E. J. Goering, Coercivity scaling in antidot lattices in $\mathrm{Fe}, \mathrm{Ni}$, and $\mathrm{NiFe}$ thin films, J. Magn. Magn. Mater. 419, 517 (2016).

[44] A. O. Adeyeye, J. A. C. Bland, and C. Daboo, Magnetic and magneto-transport behavior in variable width $\mathrm{Ni}_{80} \mathrm{Fe}_{20}$ flat wires, J. Magn. Magn. Mater. 188, L1 (1998).

[45] C. R. Pike, First-order reversal-curve diagrams and reversible magnetization, Phys. Rev. B 68, 104424 (2003). 